\section{Experimental Test of a Gravitational Effect suggested by Szekeres}

SzeKERES ${ }^{1}$ recently suggested, on the basis of his earlier work ${ }^{2}$, an interaction that causes an electromagnetic wave to change its frequency while travelling through a combination of electromagnetic and gravitational fields. The work of ref. 1 was initiated by experiments by Sadeh et al..$^{3,4}$. In one of these experiments the $21 \mathrm{~cm}$ absorption line from Taurus $A$ was observed. A decrease in the frequency of the line was found to occur when the line of sight approached the Sun. In the second experiment, a decrease in the frequency of a terrestrial source was observed which was roughly proportional to distance. Sadeh suggested that this effect was a red-shift of an electromagnetic wave while it travelled through a gravitational field (an effeet that cannot be accounted for by general relativity), while Szekeres suggested that it was a combined effect of the gravitational field and the transverse electric field of the Earth, and was dependent on direction. Szekeres's suggestion is strengthened by evidence that the effect was not observed in two-way experiments.

The hypothesis by Szekeres can be checked in the laboratory. If in a two-beam interferometer a transverse electric field is applied on one of the beams, a beat should appear in the interference pattern. If an electric field $E \mathrm{Vm}^{-1}$ is applied along a path $L m$ long, the relative change in frequency should be

$$
\frac{\Delta f}{f}=2 \times 10^{-20} E L
$$

In the suggested experiment (the light beam travelling only in one direction in the electric field) we should thus observe a beat frequency

$$
\Delta f=2 \times 10^{-20} f E L
$$

In an experiment which $\mathrm{I}$ have performed using $\mathrm{He}-\mathrm{Ne}$ laser light $\left(f \simeq 5 \times 10^{14} \mathrm{~Hz}\right)$, and a transverse electric field of $7.5 \times 10^{5} \mathrm{Vm}^{-1}$ along a path of $46 \mathrm{~cm}$, the beat frequency should be $\Delta f \simeq 3.5 \mathrm{~Hz}$.

The result shows that $\Delta f<0.2 \mathrm{~Hz}$ and possibly $\Delta f<10^{-5} \mathrm{~Hz}$ (a more detailed account will be published later). This suggests that the Sadeh experiment cannot be explained by the Szekeres hypothesis, or that the effect also depends on the frequency of the electromagnetic wave, and is stronger for long wavelengths.

J. SHAmir

Department of Physics,

Technion-Israel Institute of Technology,

Haifa, Israel.

Received February 19, 1969

${ }^{1}$ Szekeres, G., Nature, 220, 1116 (1968).

${ }^{2}$ Szekeres, G., J. Math. Mech., 6, 471 (1957).

${ }^{3}$ Sadeh, D., Knowles, S. H., and Yalpee, B. S., Seience, 159, 307 (1968).

4 Sadeh, D., Knowles, S., and Au, B., Science, 161, 567 (1968).

\section{Time-symmetric Electrodynamics and Self Action}

RECENTLY I proposed ${ }^{1}$ a mechanism for spontaneous emission as an alternative to that given by Hoyle and Narlikar ${ }^{2}$. In view of the points since raised by them ${ }^{3}$, it seems worthwhile to elaborate slightly further on the proposed theory and indicate the reason for its introducticn.

The Hoyle-Narlikar theory, because it is based on absorber theory, automatically excludes any possible effects of the electromagnetic zero point oscillations. Surely the justification for ignoring possible effects of these oscillations on the emission rate does not lie in whether they do or do not occur in a particular theory, but whether or not they exist in nature. Whether or not we believe these oscillations to exist depends on there being possible satisfactory alternative explanations of such effects as the Casimir force, verified experimentally by Deriagin and Abrikosova, which is independent of the charge (or coupling constant) $e$, rather than of effects such as the charge-dependent Lamb shift ${ }^{4}$. If these oscillations do exist, and have some effect, their existence should be recognized in any physical explanation of spontaneous emission.

Conventional quantum electrodynamics does not offer a physical explanation of spontaneous emission, it merely provides a formula for calculating the emission rate. (In fact it is quite simple to extract from the fundamental equations, which are basically time-symmetric, a formula for a non-zero rate of spontaneous absorption.) Until now, the only explanation of the cause, or stimulation, of spontaneous emission seems to have been in terms of zero point oscillations, but the conventional expression of this mechanism predicts only one half of the observed rate.

An important advantage in the work of Hoyle and Narlikar is that their approach gives a good physical explanation which predicts the correct emission rate. Unfortunately, if zero point oscillations are real, a modification to the mechanism suggested by Hoyle and Narlikar is necessary. The modification $I$ have proposed ${ }^{1}$ uses the $^{2}$ normally accepted association of zero-point oscillations with classical self-action.

The relationship of absorber theory to the theory I suggested on which this newly proposed mechanism might be based is that both theories are members of an infinite morphological class of theories formed by making the conventionally accepted postulates concerning emission and absorption intrinsically time-symmetric. These conventional postulates may be written as: $(a)$ a body can undergo an emissive transition by means of its own self action (that is, a body can emit radiation whether or not another body is present to absorb this radiation), and $(b)$ a body undergoes absorptive transitions only by means of the action (direct or indirect) of another body. Now $(a)$ and $(b)$ fit observed facts, but have the disadvantage that the arrow of time is introduced ad hoc. To remove this difficulty we can make these postulates time-symmetric (that is, make the absorption process and the emission process time inverses of each other), impose time-asymmetric boundary conditions such as a given cosmological model, and then hope to recover results consistent with observed time-asymmetric phenomena. One possible method of making the postulates time-symmetric would be to remove postulate $(a)$ and, instead, make emission the time inverse of absorption as described by $(b)$. This would lead to absorber theory. Another member of this class of theories would be obtained by replacing postulate (b) for absorption by the time inverse of $(a)$. We might call such a theory "emitter theory" by analogy. The theory which I have actually proposed is the class member midway between these two extremes, and is formed by making one half of both emission and absorption conform to $(a)$ and its time inverse, and the other half to $(b)$ and its time inverse.

The real value of this proposed theory arises because, while being dependent on cosmology, it does not rely on one particular cosmological model and will still provide an explanation for spontaneous emission if the steady state theory is eventually disproved. This is apart from the retention by the theory of the concept of electromagnetic mass, which seems important from high-energy scattering experiments ${ }^{5}$.

In conclusion, it is important to realize that various cosmological boundary conditions will produce the correct arrow of time when used in conjunction with only certain members of the class of intrinsically time-symmetric electrodynamic theories. In this light, it appears that 